\title{
Fabrication of Porous Silicon Based Humidity Sensing Elements on Paper
}

\author{
Tero Jalkanen, ${ }^{1}$ Anni Määttänen, ${ }^{2,3}$ Ermei Mäkilä, ${ }^{1}$ Jaani Tuura, ${ }^{1}$ Martti Kaasalainen, \\ Vesa-Pekka Lehto, ${ }^{4}$ Petri Ihalainen, ${ }^{2,3}$ Jouko Peltonen, ${ }^{2,3}$ and Jarno Salonen ${ }^{1,5}$ \\ ${ }^{1}$ Department of Physics and Astronomy, University of Turku, 20014 Turku, Finland \\ ${ }^{2}$ Laboratory of Physical Chemistry, Åbo Akademi University, Porthansgatan 3-5, 20500, Turku, Finland \\ ${ }^{3}$ Center for Functional Materials, Ảbo Akademi University, 20500 Turku, Finland \\ ${ }^{4}$ Department of Applied Physics, University of Eastern Finland, P.O. Box 1627, 70211 Kuopio, Finland \\ ${ }^{5}$ Turku University Centre for Materials and Surfaces (MatSurf), University of Turku, 20014 Turku, Finland \\ Correspondence should be addressed to Anni Määttänen; anni.maattanen@abo.fi and Jarno Salonen; jarno.salonen@utu.fi
}

Received 12 November 2014; Revised 20 March 2015; Accepted 23 March 2015

Academic Editor: Jesus Corres

Copyright (C) 2015 Tero Jalkanen et al. This is an open access article distributed under the Creative Commons Attribution License, which permits unrestricted use, distribution, and reproduction in any medium, provided the original work is properly cited.

A roll-to-roll compatible fabrication process of porous silicon $(\mathrm{pSi})$ based sensing elements for a real-time humidity monitoring is described. The sensing elements, consisting of printed interdigitated silver electrodes and a spray-coated pSi layer, were fabricated on a coated paper substrate by a two-step process. Capacitive and resistive responses of the sensing elements were examined under different concentrations of humidity. More than a three orders of magnitude reproducible decrease in resistance was measured when the relative humidity (RH) was increased from $0 \%$ to $90 \%$. A relatively fast recovery without the need of any refreshing methods was observed with a change in RH. Humidity background signal and hysteresis arising from the paper substrate were dependent on the thickness of sensing pSi layer. Hysteresis in most optimal sensing element setup (a thick pSi layer) was still noticeable but not detrimental for the sensing. In addition to electrical characterization of sensing elements, thermal degradation and moisture adsorption properties of the paper substrate were examined in connection to the fabrication process of the silver electrodes and the moisture sensitivity of the paper. The results pave the way towards the development of low-cost humidity sensors which could be utilized, for example, in smart packaging applications or in smart cities to monitor the environment.

\section{Introduction}

Printed electronics has been proposed as a viable option for manufacturing low-end products on large areas of flexible substrates [1]. Utilization of roll-to-roll (R2R) compatible fabrication methods enables large scale preparation of flexible devices with extremely low production costs. Devices, such as transistors, have been prepared on flexible substrates from several materials [2-6]. One interesting emerging application is the fabrication of flexible gas sensors [7-11]. In particular, low-cost printed flexible sensors could be utilized in smart packaging applications for monitoring perishable goods, such as food, or in smart cities to monitor the environment [12]. Unfortunately, in many cases the fabrication of flexible sensors requires multiple production steps that are often difficult to upscale and incompatible with R2R printing methods.
This leads to increased production costs often making large scale production unrealistic, as the cost for printing scales at least linearly with the number of fabrication steps [13]. Lowcost fabrication can be achieved via utilization of different printing methods and minimization of printing steps. Printed gas sensors on flexible substrates have been demonstrated with materials such as polyaniline $[7,14]$, carbon nanotubes [8], reduced graphene oxide $[15,16]$, and copper acetate [17].

In addition to the active sensing material, the properties of the substrate also play a large role in the fabrication and the properties of flexible sensors. Plastic and paper are common choices as substrate materials and are suitable for large scale R2R printing. From the viewpoint of recyclability and sustainable development, paper substrates would be more preferable than plastic ones $[6,18]$. Paper is also considerably cheaper than plastic and can better withstand 
heating steps, such as thermal annealing or IR sintering, often used in R2R printing processes [18-20]. However, the inherent surface roughness and porosity make device fabrication on paper more complicated, and for this reason the majority of flexible devices have been constructed on plastic substrates. To circumvent this problem, the paper substrate can be coated with plastic, such as polyethylene [21], making device fabrication more feasible but at the same time compromising the recyclability of the substrate. Another way to improve surface properties of paper is to coat it with binders and pigments $[6,18,22]$. For example, by multilayer coating of the base substrate with successive latex and mineral pigment composite layers, a recyclable substrate with sufficient printing and barrier properties has been fabricated [6]. Reduced surface roughness and porosity make this type of a substrate suitable for printed electronics [23]. Moreover, the coffee-stain effect is not as prominent on paper and printed structures show improved adhesion (without the need of any surface treatment) compared to plastic or glass substrates $[20,23,24]$. The benefit with the fabrication of printed flexible circuits with a sensing element whose properties can be changed according to the application is that the circuits enable remote reading of the sensor with a RFID tag (radio-frequency identification) $[25,26]$.

Recently, we demonstrated the feasibility of solutionprocessable porous $\mathrm{Si}(\mathrm{pSi})$ particles for producing printed sensors, by preparing humidity sensors on a glass substrate [27]. A pSi is an interesting material for sensing applications, because it hosts a large internal surface area, and the surface chemistry can be modified to accommodate for a large number of environmental and biochemical sensing schemes $[28,29]$. In addition, with proper surface treatments the Si surface can be made more stable compared, for example, to conducting polymers whose properties are often subject to change upon aging $[30,31]$.

In the present work, we expand on our previous work and demonstrate R2R compatible two-step fabrication process for obtaining a sensing element for monitoring relative humidity. In the first step, interdigitated silver electrode patterns were printed on the multilayer coated paper substrate. This was followed by deposition of solution-processable pSi particles on top of the electrode pattern by spray-coating. The deposited pSi acts as a sensing layer that enables capacitive or resistive sensing between the electrodes. The electrical characteristics of the sensing elements were determined, and the sensing properties were evaluated by exposing the elements to varying concentrations of $\mathrm{RH}$. The thermal stability of paper substrates was evaluated because when these devices are fabricated in a $\mathrm{R} 2 \mathrm{R}$ process the paper substrates are exposed to high temperatures for short times, for example, during the sintering of the silver electrodes into conductive electrodes. Finally, the barrier properties of the substrate were assessed with gravimetric humidity sorption measurements.

\section{Materials and Methods}

2.1. Sensing Element Fabrication on Coated Paper. Freestanding pSi films were produced with electrochemical anodization, as described in our previous work [27]. The as-anodized pSi films were stabilized with a two-step thermal carbonization process, as described previously [32, 33]. Briefly, a $10 \mathrm{~min}$ thermal hydrocarbonization treatment was conducted under $\mathrm{N}_{2}$ /acetylene $(1: 1)$ flow at $500^{\circ} \mathrm{C}$, followed by a thermal treatment at a higher temperature of $820^{\circ} \mathrm{C}$ resulting in a stable and hydrophilic surface.

The pSi micro- and nanoparticles were produced from the stabilized free-standing pSi films by means of high energy planetary ball milling in an ethanol solution. The high porosity layers in the free-standing multilayer films act as fracture planes, and the particle size can be controlled by adjusting the thickness of the low porosity mesoporous layer [27]. Different particle size fractions were separated by sieving and centrifugation. The resulting pSi particles were dispersed in a toluene suspension, making them solution processable. A broad particle size distribution ( $100-2000 \mathrm{~nm})$ was used to improve packing and adhesion of the silicon film on the substrate [27].

A multilayer coated paper (fabricated using either reverse gravure coating in laboratory [6] or industrial pilot-sized curtain coater [34]) was used as a substrate. Different interdigitated silver electrode patterns with varying gaps (100$500 \mu \mathrm{m}$ ) were produced on the substrate with two techniques, namely, flexography and inkjet printing. Flexographically printed interdigitated electrodes were R2R fabricated with a custom-built hybrid printed, as previously described, using a silver ink (125-06, Creative Materials Inc.) [35]. Inkjet printing was carried out using a piezoelectric Dimatix Materials Printer (DMP-2831, Dimatix-Fujifilm, Inc.) and a replaceable cartridge (DMC-11610) with a $10 \mathrm{pL}$ nominal drop volume. The electrodes were printed using a nanoparticle based silver ink (SunTronic, U5603, $20 \mathrm{wt} . \%$ ) and a drop spacing of $20 \mu \mathrm{m}$. For obtaining conducting electrodes, both the flexographically and inkjet-printed silver electrodes were IR sintered [35]. The pSi particle solution was deposited on top of the electrode configuration with a simple spray coater (Clas Ohlson, Finland). All fabrication steps took place under ambient atmospheric conditions under a fume collector.

\subsection{Characterization Instrumentation. Hygroscopicity Mea-} surement Apparatus (HMA) (PuuMan Oy) was used for determining sample weight increase due to water sorption under different $\mathrm{RH}$ conditions at room temperature $\left(26^{\circ} \mathrm{C}\right)$. Thermal analysis on paper and plastic substrates was conducted with differential scanning calorimetry (DSC) (Diamond DSC, PerkinElmer Inc.) and simultaneous thermal analyzer (STA) (STA 6000, PerkinElmer Inc.). Calibration of the instruments was carried out with indium and silver. The measurements were conducted in an atmosphere of synthetic air with a heating rate of $10^{\circ} \mathrm{C} / \mathrm{min}$ (for DSC) and $20^{\circ} \mathrm{C} / \mathrm{min}$ (for STA). The pSi particle size distribution was determined with dynamic light scattering measurements (Zetasizer Nano ZS, Malvern Instruments Ltd.).

An NTEGRA Prima (NT-MDT, Russia) atomic force microscope (AFM) was used for analyzing the thickness of the spray-coated pSi layers. The images were scanned in ambient conditions $\left(T=24 \pm 2^{\circ} \mathrm{C}, \mathrm{RH}=29 \pm 2 \%\right)$ operating with intermittent-contact mode at the repulsive regime using rectangular cantilevers (NSG10, NT-MDT, Russia) with a 


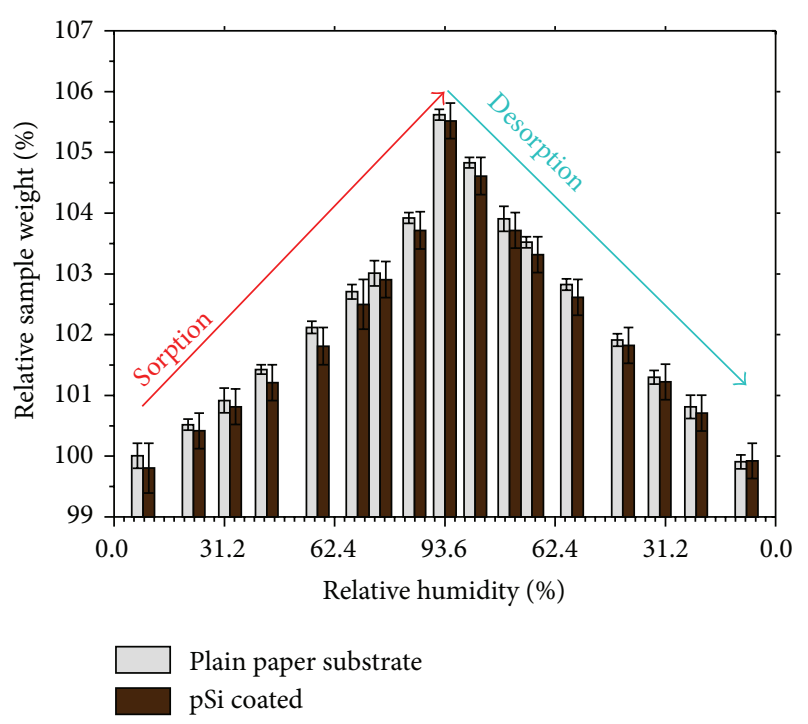

FIGURE 1: Plain paper substrate and a substrate coated with pSi both exhibit an equally large humidity related weight increase.

$0.18 \mathrm{~Hz}$ scan rate. The images were processed and analyzed using SPIP (Scanning Probe Image Processor, Image Metrology, Denmark) software.

2.3. Sensing Experiments. The prepared sensing elements were placed inside an atmospheric chamber which was connected to a gas line. Nitrogen was used as a carrier gas and digital mass flow controllers (5860S, Brooks) were used to control gas flow to the chamber. Atmospheric composition was changed by diverting controlled portions of the nitrogen carrier flow through a water container [27]. A humidity measurement probe (HMP35E, Vaisala, accuracy of $\pm 3 \% \mathrm{RH}$ at a humidity range of $90-100 \%$ ) was used for validating the RH values. Electrical parameters were measured by connecting the electrodes of the sensor to a LCR Meter (1920 Precision, QuadTech).

\section{Results and Discussion}

3.1. Substrate Properties. Paper is intrinsically porous and hygroscopic and the sorbed moisture may become a source for inconsistent sensor response. In order to investigate the magnitude of humidity sorption to the substrate, the weight increase of several samples was evaluated in different humidity conditions. Figure 1 compares a plain paper substrate and one that is spray-coated with the pSi layer. In both cases, the moisture uptake increased quite linearly as a function of $\mathrm{RH}$ up to $\mathrm{RH}$ of $85.0 \%$ beyond which a relatively large increase in the uptake was observed. This is consistent with a typical moisture uptake behavior of initially dry paper when exposed to increasing humidity $[36,37]$. When the humidity level was gradually raised to $93.6 \mathrm{RH} \%$ the relative weight of both samples increased approximately to $105.5 \%$. When the $\mathrm{RH}$ value was lowered back to the initial value, in a stepwise manner, clear hysteresis was observed throughout the entire range. The actual equilibrium moisture content present in a particular paper at a specific $\mathrm{RH}$ is determined by the type, purity, and quantity of fibers, type and quantity of filler, nature and quantity of adhesive and binders, and degree of fiber treatment. In addition, the past moisture history of the paper (initially dry or wet) will affect the equilibrium moisture content, and different moisture uptake in sorption isotherm is observed whether equilibrium moisture content is approached through wetting of initially dry paper or through drying of wetted paper [37]. The difference between the humidity sorption behavior in the plain paper and the $\mathrm{pSi}$ coated samples was negligible. This is to be expected since the $\mathrm{pSi}$ layer is quite thin, thus constituting an insignificant portion of the total sample mass. In comparison, a clear weight increase of $4 \%$ was observed also for a plain Scotch tape sample. This shows that, in order to diminish the sorption of humidity and for attaining long-term sensor stability and smaller hysteresis, a careful selection of coating materials and barrier coating on the backside of the substrate is important. Good barrier properties against humidity would prevent gas absorption to the porous fiber base of the paper, thus reducing the undesirable leakage currents travelling through the substrate.

Thermal analysis was conducted for polypropylene, Mylar A, regular fine paper, and the multilayer coated paper [6]. The results are shown in Figure 2. It is obvious from the DSC curves (Figure 2(a)) that the characteristics of regular fine paper and the special multilayer coated paper are quite similar. Both substrates exhibit a broad endothermic hump during the first heating run, which is associated with desorption of moisture. The only difference in the DSC curves measured for the paper substrates is the exothermic peak exhibited by the multilayer coated paper substrate around $200^{\circ} \mathrm{C}$. This peak is not seen during the second heating cycle. The same peak is also present in the heat flow curve measured with STA (Figure 2(b)). However, from the TG curve one can see that it is not accompanied with weight loss (Figure 2(c)). This suggests that the peak is most likely connected to a recrystallization process of one of the materials used for coating the paper, possibly kaolin $[38,39]$. A similar exothermic dip is found from the first heating curve, when DSC measurements are performed on kaolin powder (Figure 2(d)). The broad endotherm is attributed to desorption of moisture from the powder sample (Figure 2(d)).

Based on the results shown in Figure 2, it is clear that the multilayer coated substrate presents only limited thermal events and no thermal degradation, as temperatures approach $300^{\circ} \mathrm{C}$. The onset temperature for the first large exothermic peak (Figure 2(b)) determined as an average of four paper samples was $T_{\text {onset }}=342.9 \pm 1.4^{\circ} \mathrm{C}$. This peak is accompanied with significant weight loss (Figure 2(c)). The onset temperature is significantly higher than the melting temperature observed for the Mylar A substrate $\left(T_{m}=\right.$ $253.0 \pm 0.6^{\circ} \mathrm{C}$ ), which, in turn, is considerably higher than that of many other inexpensive plastic substrates, such as polypropylene (Figure 2(a)). Based on the thermal analysis, the multilayer coated paper substrate can be considered thermally more stable than inexpensive plastic substrates. A good thermal stability of the substrate is important considering that, for example, the solvents and the stabilizing capping 


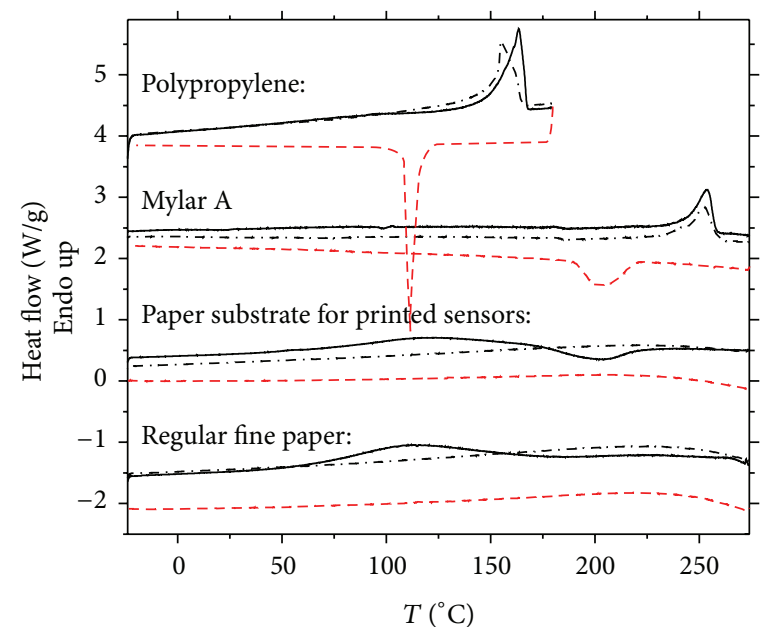

(a)

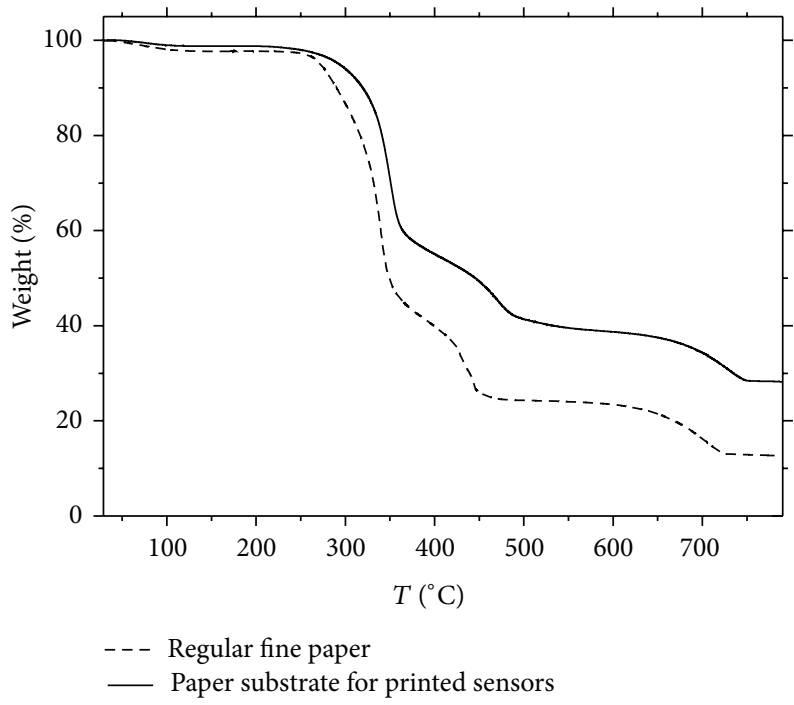

(c)

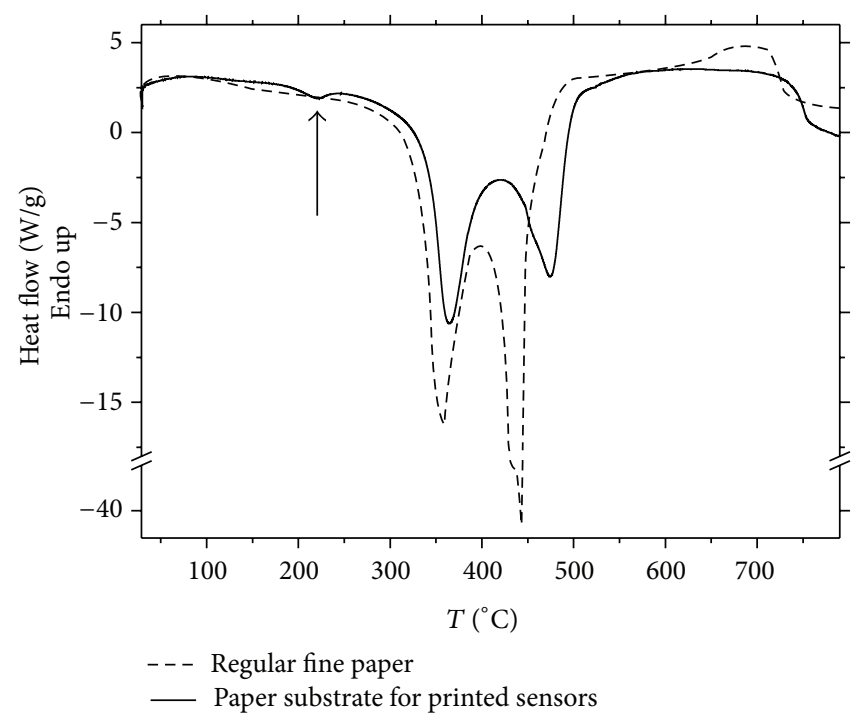

(b)

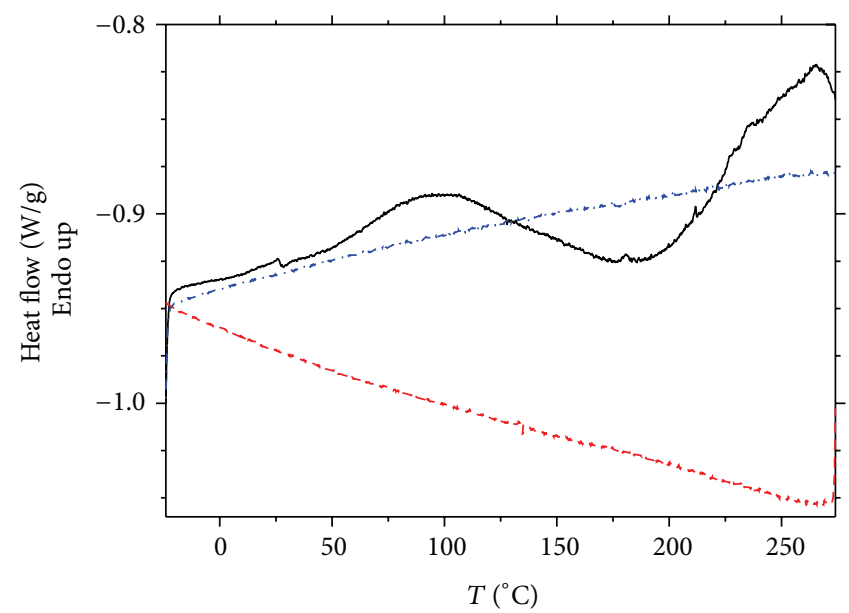

Kaolin powder:

_ First heating cycle
- - Cooling cycle
-_ Second heating cycle

(d)

Figure 2: (a) DSC curves for four different flexible substrates. The solid curves were measured during the first heating cycle, and the dashed red curves were measured during cooling. The dotted dashed curves were measured for the second heating cycle. STA results for the two paper substrates: (b) heat flow curves and (c) the corresponding TG curves. The arrow in the heat flow curve points out the exothermic peak that is present only in the curves measured for the multilayer coated paper substrate. (d) Similar exothermic dip is also found from the first heating cycle measured for kaolin powder with DSC.

agents used in the nanoparticle based inks are preferentially totally removed during the sintering process for obtaining the highest possible conductivity and purity of the electrodes. In case of the AgNP-based ink used in this study, the $20 \mathrm{wt} . \%$ solid content value was achieved at a $200^{\circ} \mathrm{C}$ temperature in a TGA experiment [40]. Higher temperatures may be required for other encapsulation materials [41, 42].

3.2. Initial Electrical Characterization and Performance of the Sensing Elements. Initial electrical characterization and performance tests were carried out using spray-coated $\mathrm{pSi}$ layers on flexographically printed electrodes (Figure 3(a)). In addition Figure 3(b) shows the R2R fabrication of sensing elements with a custom-built hybrid printer [34].

In order to determine the operational measurement frequency range for the sensing element, susceptance was measured as a function of frequency for two different $\mathrm{RH}$ values (Figure 4). Susceptance $B_{p}$ for an equivalent parallel circuit consisting of a resistor and a capacitor can be determined as

$$
B_{p}=-\frac{X_{s}^{2}}{R_{s}^{2}+X_{s}^{2}}
$$




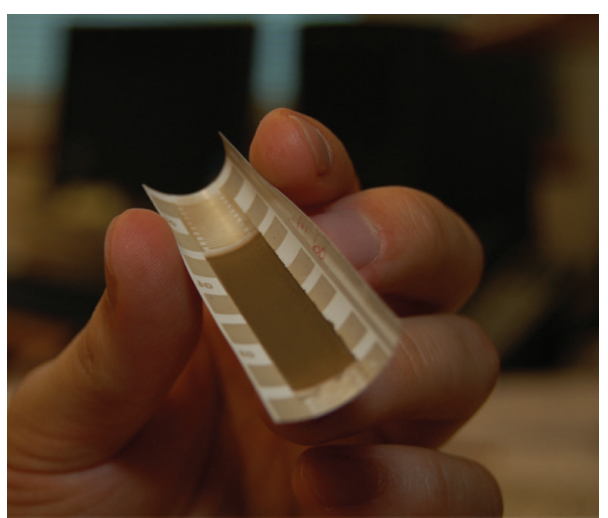

(a)

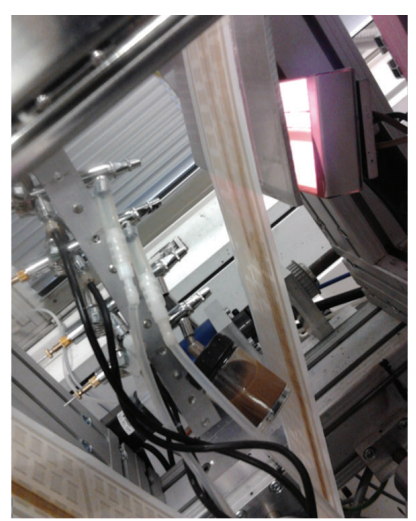

(b)

FIGURE 3: A photograph showing (a) a spray-coated pSi layer on flexographically printed silver electrodes and (b) the process of R2R spraycoating of pSi layer and online IR drying using the custom-build hybrid printer.

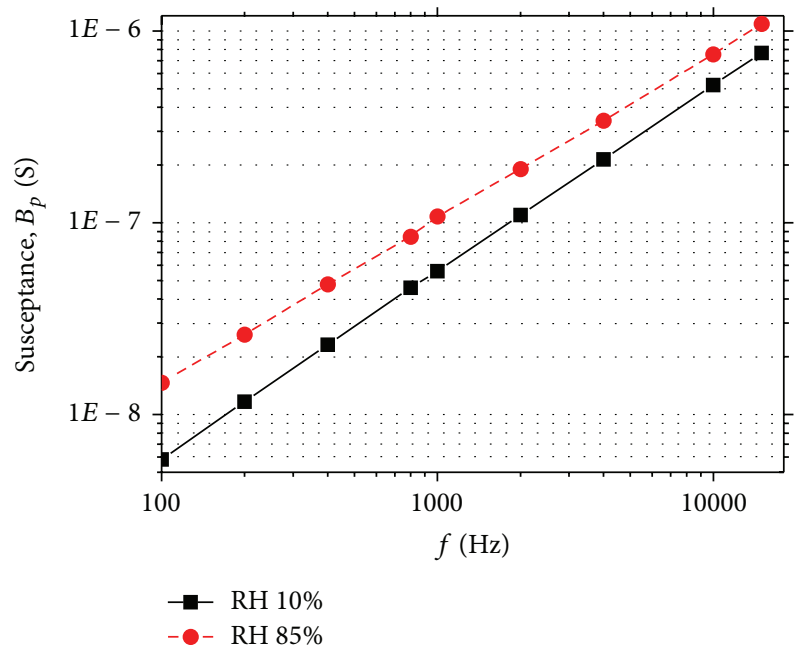

FIGURE 4: The susceptance curve shows that the sensing elements can be operated on a broad frequency range.

where $X_{s}$ and $R_{s}$ are the reactance and the resistance for a corresponding equivalent series circuit, respectively. Based on Figure 4, the sensing elements are operational over a broad frequency range. Sensitivity was higher for smaller frequencies. However, the smallest frequencies displayed significantly higher noise levels. Therefore, a measurement frequency value of $1 \mathrm{kHz}$ was chosen for the sensing experiments. This value provided the best signal to noise ratio, thus resulting in optimal response. A measurement voltage of $1 \mathrm{~V}$ was used for the experiments. Slight differences in the measured susceptance values were observed for the different electrode designs, but the overall shape of the respective curves retained a similar form. In addition to electrode design, the thickness of the spray-coated pSi layer also affects the numerical values obtainable for susceptance.

Figure 5(a) shows the relative capacitive response to different RH levels. The response increased in accordance with concentration, and for concentrations above RH 50\% the sensitivity was below $5 \mathrm{RH} \%$; that is, a concentration chance that is smaller than $5 \mathrm{RH} \%$ can be detected. In the beginning of the measurement (Figure 5(a)), two consecutive high humidity pulses (RH 95\%) were created. A slight variation between the respective response curves was detected. As this can be caused by concentration fluctuations in the testing chamber for high $\mathrm{RH}$ values, the reproducibility of the response was tested with lower concentrations. Figure 5(b) displays the response to four consecutive $85 \mathrm{RH} \%$ pulses. Reproducible results were obtained, albeit with small differences between the pulses (about 5\% between the first and the last pulse). However, the variation between the consecutive pulses is clearly smaller than that for the RH 95\% pulses. It should also be noted that the signal recovery back to the zerolevel occurred quickly, even though no refreshing methods were employed. Figure 5(a) also shows the response measured for the paper substrate with just the electrode pattern without the sensing layer. As can be seen, the response is considerably increased with the addition of the sensing layer. Compared to the nearly linear humidity response of the plain paper, an increased sensitivity was obtained at increasing humidity conditions with the sample that contained the pSi layer. This is a consequence of capillary condensation that occurs when adsorbed vapor at the numerous nanometer range pores in the sensing material turns to liquid [43]. It should be noted though that the thickness of the sensing layer plays a crucial role in determining the difference in sensing capabilities.

Comparison of Figures 5(a) and 5(b) shows an interesting phenomenon. A considerably more sensitive response (about tenfold) is seen in Figure 5(b) as a consequence to subjection to a $\mathrm{RH} 85 \%$ pulse. This is a direct consequence of the bottom contact electrode design that was used. Therefore, sensing is linked to gas diffusion through the pSi layer, which means that differences in layer thickness lead to variation in sensitivity and response time alike. Printing the electrode design on top of the sensing layer would presumably minimize this effect, but unfortunately the rough and porous surfaces of the sensing layer result in inadequate print quality, thus making this approach unviable. Consequently, the thickness of the pSi layer should be optimized, in order to obtain the best possible sensitivity and response time. It is probable that 


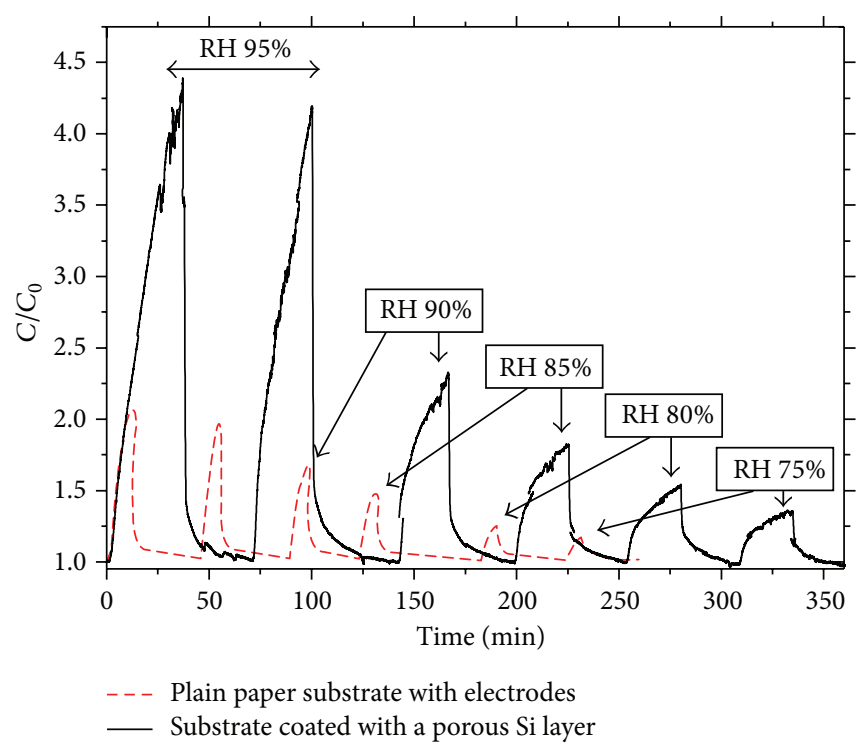

(a)

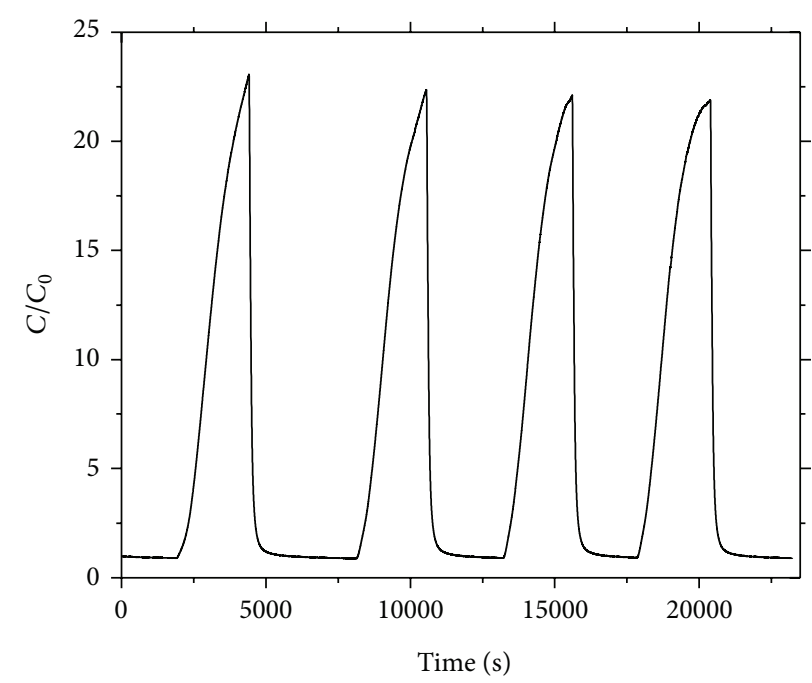

(b)

FIGURE 5: (a) Relative capacitive response to changes in RH concentration shows that different concentrations can be clearly distinguished and that with proper thickness of the sensing layer the signal from the sensing layer is clearly stronger than the signal from the substrate. (b) Four consecutive RH 85\% pulses demonstrate that the response is quite consistent, providing reproducible results.

a trade-off between the two occurs at some crucial layer thickness, where thinner layers result in faster response but decreased sensitivity. In addition, optimization of the coating process is very important when large scale fabrication with a R2R process is considered (Figure 3(b)). Even though large quantities can be prepared when R2R production is used, differences in the sensing capability of an individual sensing element can be large if the thickness of the pSi layer is not carefully monitored.

3.3. Optimizing the Thickness of the Sensing Layer towards Humidity Response. The effect of the thickness of the spraycoated pSi layer towards humidity response was studied using sensing elements fabricated on inkjet-printed silver electrodes (Figure 6). Three different amounts of pSi were applied on the electrodes: $0.3 \mathrm{mg}, 0.6 \mathrm{mg}$, and $1 \mathrm{mg}$, and their resistive response in the whole $\mathrm{RH}$ range was studied. In addition, the sensing layers with the lowest (Figure 6(b)) and the highest (Figure 6(d)) pSi coating amounts were selected for more detailed analysis by optical microscope and AFM (Figure 7). The optical micrograph of the sample with the lowest coating amount showed that the inkjet-printed silver finger electrodes were still at some spots reflecting similarly to the uncoated silver contacts (Figure 7(a)). This suggested that the sensing layer was not fully covering the electrodes. The AFM topograph further supported the above conclusion, showing that in some places the pSi layer either was totally absent or was hard to distinguish from the paper substrate (Figure $7(\mathrm{c})$ ). With the highest coating amount, the silver fingers were visible but appeared much darker and nonreflecting, suggesting that the interdigitated fingers were fully covered by a thicker pSi layer (Figure 7(b)). In addition, AFM topograph showed a fully covering Si layer which was clearly distinguishable from paper substrate (Figure 7(d)).
Figure 8 shows AFM height line profiles obtained at the border of paper substrate and pSi layer for the samples with lowest $(0.3 \mathrm{mg})$ and highest $(1 \mathrm{mg}) \mathrm{pSi}$ coating amounts. For the former, the pSi layer thickness varied between 0 and $1500 \mathrm{~nm}$, the average being clearly below $500 \mathrm{~nm}$ (Figure $8(a))$. The thickness variations were in accordance with the particle size distribution. However, the average thickness of the pSi layer was difficult to determine accurately due to the relatively high roughness of the paper substrate. The height variations in the sample with highest pSi coating amount were between 500 and $2500 \mathrm{~nm}$, the average being about $750 \mathrm{~nm}$ (Figure 8(b)).

Figure 9 displays resistive response in the whole $\mathrm{RH}$ range using the sensing element with the highest pSi coating amount $(1 \mathrm{mg})$. The resistive responses of the sensing elements with the other coating amounts (i.e., $0.3 \mathrm{mg}$ and $0.6 \mathrm{mg}$ ) were not significantly higher compared to the background response arising from the paper substrate (data not shown). Thus, the sensing element with the thickest $\mathrm{pSi}$ layer can be considered the most optimal setup tested in this study. In addition, a significant and nearly identical hysteresis was observed with samples with no or very thin pSi layer. The hysteresis decreased but was still prominent for the sample with the thickest pSi layer (Figure 9). A previous study has shown that the similar sensor element design on a glass substrate exhibited negligible hysteresis in resistive response between RH 0\% and RH 90\% [27]. Thus, the observed hysteresis is not the material property but is influenced by both the thickness of the sensing layer and hydroscopic property of the substrate.

\section{Conclusions}

In conclusion, functional sensing elements based on pSi particles were produced on a paper substrate with facile 


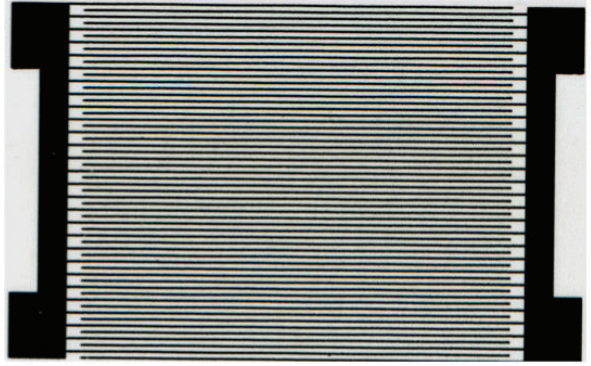

(a)

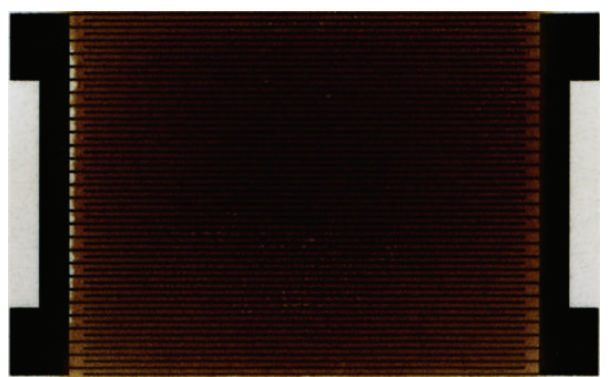

(c)

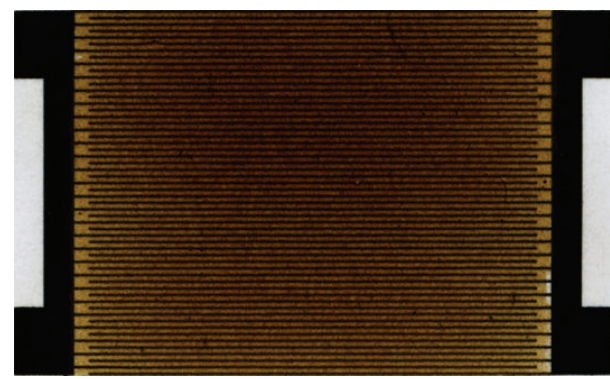

(b)

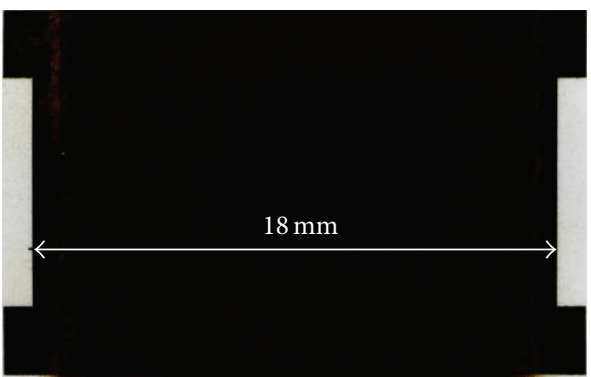

(d)

FIGURE 6: Scanned images of inkjet-printed Ag electrodes covered by (a) $0 \mathrm{mg}$, (b) $0.3 \mathrm{mg}$, (c) $0.6 \mathrm{mg}$, and (d) $1 \mathrm{mg}$ of spray-coated pSi.

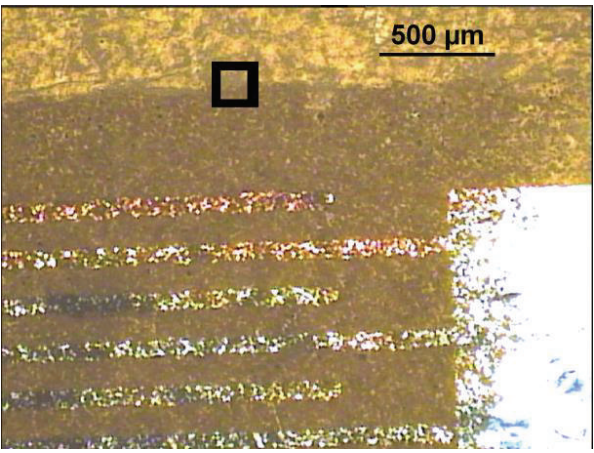

(a)

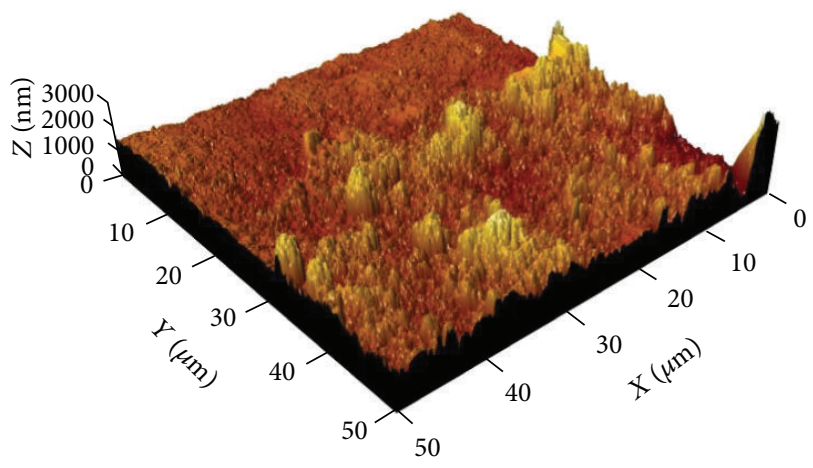

(c)

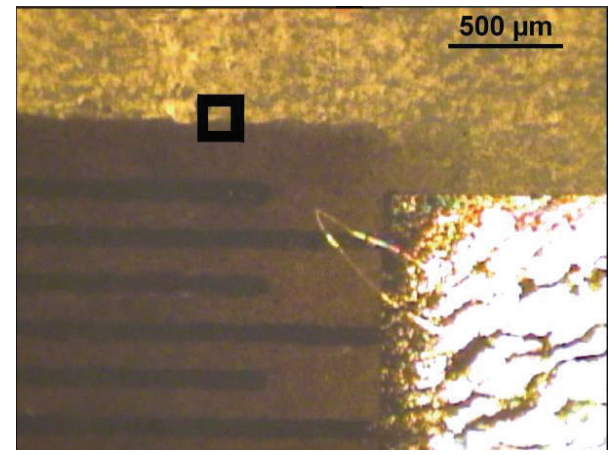

(b)

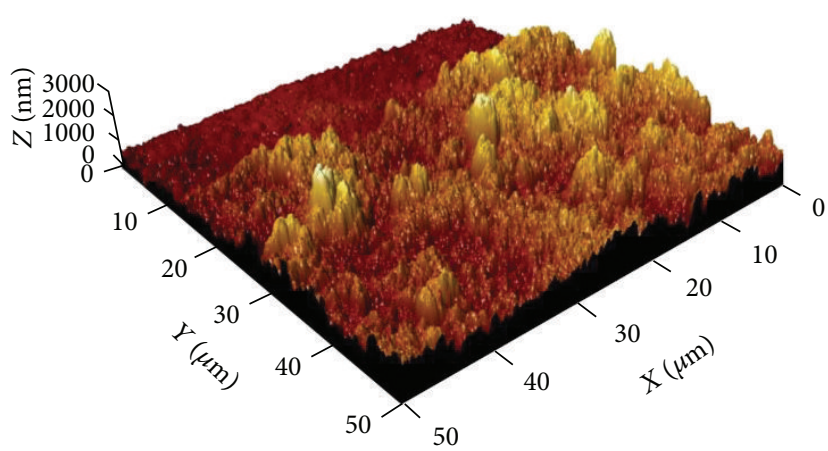

(d)

FIGURE 7: Optical micrographs of sensing element with (a) the lowest $(0.3 \mathrm{mg})$ and (b) the highest $(1 \mathrm{mg})$ amount of spray-coated pSi on the interdigitated inkjet-printed silver electrodes. Also shown are AFM topographical images $(50 \mu \mathrm{m} \times 50 \mu \mathrm{m})$ of the border of paper and (c) thin and (d) thick layer of pSi. The black squares indicate the capture spots of the AFM topographs. 


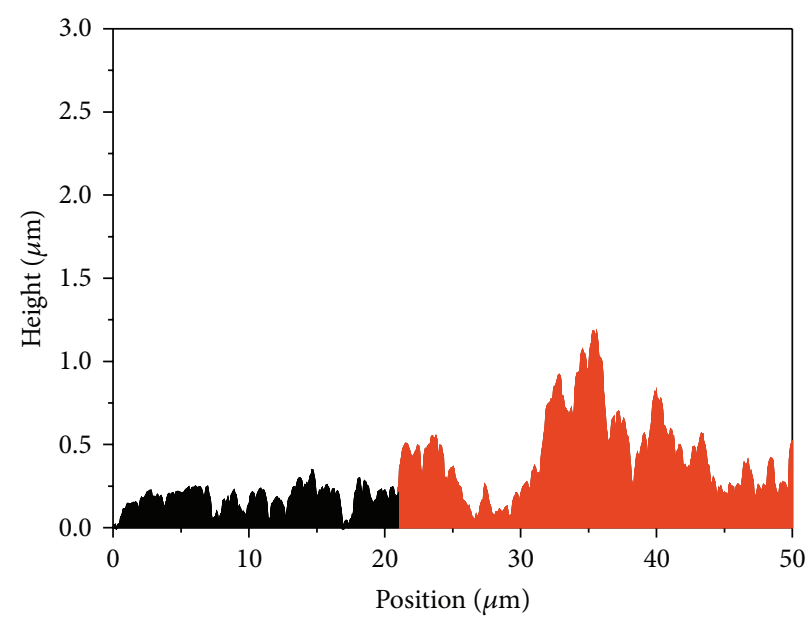

$\square$ Thin sensing layer
Substrate

(a)

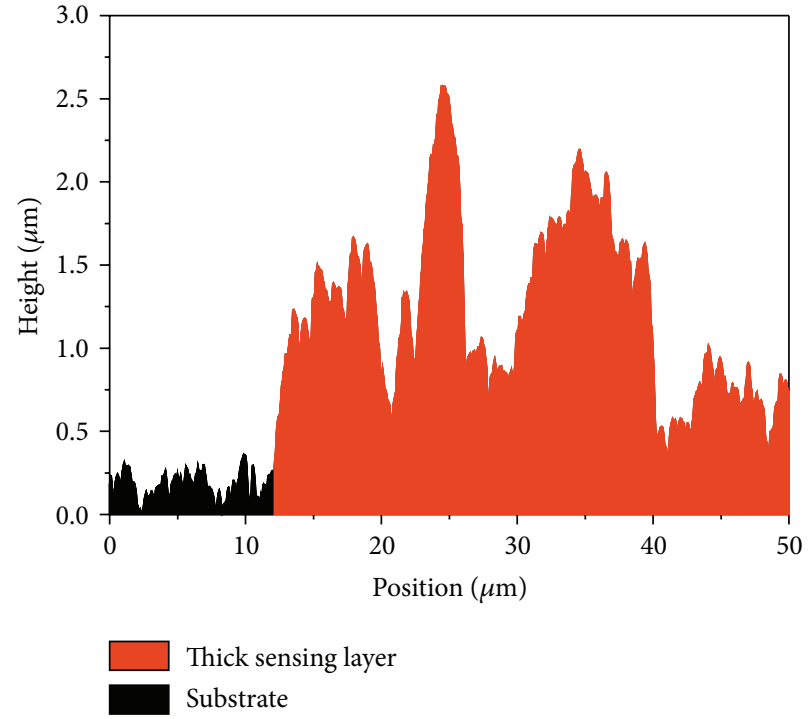

(b)

FIGURE 8: AFM height line profiles obtained at the border of the paper substrate and (a) a thin and (b) a thick pSi layer.

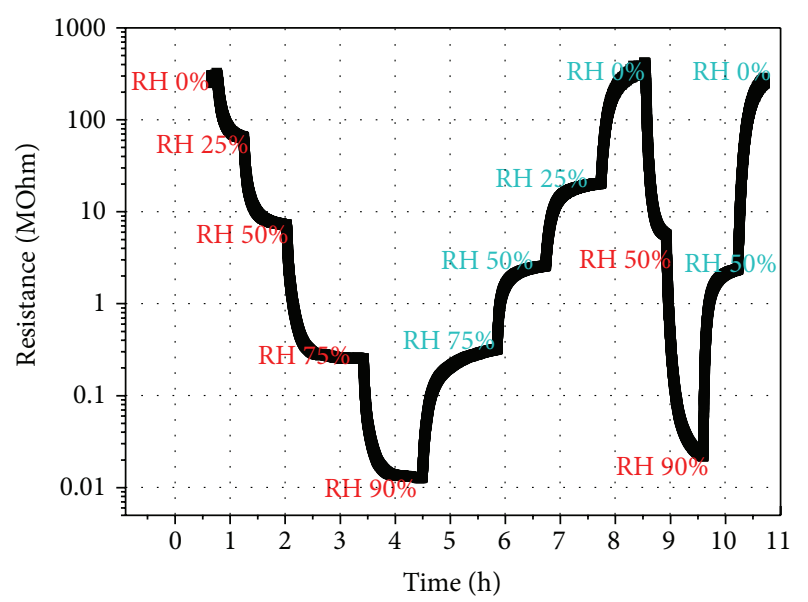

Figure 9: Resistive response between RH 0\% and RH 90\%. Clear hysteresis is observed, which is mainly attributed to unwanted humidity adsorption to the substrate.

R2R compatible methods. The robust fabrication steps are easily upscalable, which suggests that the presented method is feasible for mass production of humidity sensing devices. Reproducible results were obtained when the sensing elements were exposed to different concentrations of humidity. In addition, relatively fast recovery was observed even though no refreshing methods were employed. However, large differences in sensitivities were observed for different sensing layer thicknesses. Considerable humidity background signal and hysteresis arising from the paper substrate were dependent on the thickness of sensing pSi layer. Hysteresis in most optimal sensing element setup (a thick pSi layer) was still noticeable but not detrimental for the sensing. Both the thickness of the sensing layer and hydroscopic property of the substrate have to be considered when further developing the low-cost paper-based humidity sensors towards enhanced performance, that is, higher sensitivity and lower hysteresis.

\section{Conflict of Interests}

The authors declare that there is no conflict of interests regarding the publication of this paper.

\section{Acknowledgments}

The authors would like to thank Dr. Roger Bollström for the R2R sample preparations. This work was supported by the Finnish Funding Agency for Technology and Innovation (Tekes) under the FLEX-SENS project and by the strategic funding of University of Eastern Finland.

\section{References}

[1] M. A. M. Leenen, V. Arning, H. Thiem, J. Steiger, and R. Anselmann, "Printable electronics: flexibility for the future," Physica Status Solidi A: Applications and Materials Science, vol. 206, no. 4, pp. 588-597, 2009.

[2] M. M. Ling and Z. Bao, "Thin film deposition, patterning, and printing in organic thin film transistors," Chemistry of Materials, vol. 16, no. 23, pp. 4824-4840, 2004.

[3] U. Haas, H. Gold, A. Haase, G. Jakopic, and B. Stadlober, "Submicron pentacene-based organic thin film transistors on flexible substrates," Applied Physics Letters, vol. 91, no. 4, Article ID 043511, 2007.

[4] K. Song, J. Noh, T. Jun, Y. Jung, H.-Y. Kang, and J. Moon, "Fully flexible solution-deposited $\mathrm{ZnO}$ thin-film transistors," Advanced Materials, vol. 22, no. 38, pp. 4308-4312, 2010.

[5] K.-J. Baeg, M. Caironi, and Y.-Y. Noh, "Toward printed integrated circuits based on unipolar or ambipolar polymer semiconductors," Advanced Materials, vol. 25, no. 31, pp. 42104244, 2013. 
[6] R. Bollström, A. Määttänen, D. Tobjörk et al., "A multilayer coated fiber-based substrate suitable for printed functionality," Organic Electronics: Physics, Materials, Applications, vol. 10, no. 5, pp. 1020-1023, 2009.

[7] K. Crowley, A. Morrin, A. Hernandez et al., "Fabrication of an ammonia gas sensor using inkjet-printed polyaniline nanoparticles," Talanta, vol. 77, no. 2, pp. 710-717, 2008.

[8] S. Ammu, V. Dua, S. R. Agnihotra et al., "Flexible, all-organic chemiresistor for detecting chemically aggressive vapors," Journal of the American Chemical Society, vol. 134, no. 10, pp. 45534556, 2012.

[9] D. Briand, A. Oprea, J. Courbat, and N. Bârsan, "Making environmental sensors on plastic foil," Materials Today, vol. 14, no. 9, pp. 416-423, 2011.

[10] F. Molina-Lopez, D. Briand, and N. F. de Rooij, "All additive inkjet printed humidity sensors on plastic substrate," Sensors and Actuators B: Chemical, vol. 166-167, pp. 212-222, 2012.

[11] M. Segev-Bar and H. Haick, "Flexible sensors based on nanoparticles," ACS Nano, vol. 7, no. 10, pp. 8366-8378, 2013.

[12] A. Solanas, C. Patsakis, M. Conti et al., "Smart health: a contextaware health paradigm within smart cities," IEEE Communications Magazine, vol. 52, no. 8, pp. 74-81, 2014.

[13] M. Berggren, D. Nilsson, and N. D. Robinson, "Organic materials for printed electronics," Nature Materials, vol. 6, no. 1, pp. 3-5, 2007.

[14] T. Hibbard, K. Crowley, F. Kelly et al., "Point of care monitoring of hemodialysis patients with a breath ammonia measurement device based on printed polyaniline nanoparticle sensors," Analytical Chemistry, vol. 85, no. 24, pp. 12158-12165, 2013.

[15] V. Dua, S. P. Surwade, S. Ammu et al., "All-organic vapor sensor using inkjet-printed reduced graphene oxide," Angewandte Chemie, vol. 49, no. 12, pp. 2154-2157, 2010.

[16] J. Hassinen, J. Kauppila, J. Leiro et al., "Low-cost reduced graphene oxide-based conductometric nitrogen dioxidesensitive sensor on paper," Analytical and Bioanalytical Chemistry, vol. 405, no. 11, pp. 3611-3617, 2013.

[17] J. Sarfraz, P. Ihalainen, A. Määttänen et al., "A printed $\mathrm{H}_{2} \mathrm{~S}$ sensor with electro-optical response," Sensors and Actuators, $B$ : Chemical, vol. 191, pp. 821-827, 2014.

[18] D. Tobjörk and R. Österbacka, "Paper electronics," Advanced Materials, vol. 23, no. 17, pp. 1935-1961, 2011.

[19] B. Singh and N. Sharma, "Mechanistic implications of plastic degradation," Polymer Degradation and Stability, vol. 93, no. 3, pp. 561-584, 2008.

[20] D. Tobjörk, H. Aarnio, P. Pulkkinen et al., "IR-sintering of inkjet printed metal-nanoparticles on paper," Thin Solid Films, vol. 520, no. 7, pp. 2949-2955, 2012.

[21] P. Andersson, D. Nilsson, P. Svensson et al., "Active matrix displays based on all-organic electrochemical smart pixels printed on paper," Advanced Materials, vol. 14, no. 20, pp. 14601464, 2002.

[22] B. Trnovec, M. Stanel, U. Hahn et al., Coated Paper for Printed Electronics, Professional Papermaking, 2009.

[23] A. Määttänen, U. Vanamo, P. Ihalainen et al., "A low-cost paperbased inkjet-printed platform for electrochemical analyses," Sensors and Actuators B: Chemical, vol. 177, pp. 153-162, 2013.

[24] N. Chitvoranund, S. Jiemsirilers, and D. P. Kashima, "Effects of surface treatments on adhesion of silver film on glass substrate fabricated by electroless plating," Journal of the Australian Ceramic Society, vol. 49, no. 1, pp. 62-69, 2013.
[25] R. A. Potyrailo, N. Nagraj, Z. Tang, F. J. Mondello, C. Surman, and W. Morris, "Battery-free radio frequency identification (RFID) sensors for food quality and safety," Journal of Agricultural and Food Chemistry, vol. 60, no. 35, pp. 8535-8543, 2012.

[26] X. Wang, O. Larsson, D. Platt et al., "An all-printed wireless humidity sensor label," Sensors and Actuators B: Chemical, vol. 166-167, pp. 556-561, 2012.

[27] T. Jalkanen, E. Mäkilä, A. Määttänen et al., "Porous silicon micro- and nanoparticles for printed humidity sensors," Applied Physics Letters, vol. 101, no. 26, Article ID 263110, 2012.

[28] J. M. Buriak, "Organometallic chemistry on silicon and germanium surfaces," Chemical Reviews, vol. 102, no. 5, pp. 1271-1308, 2002.

[29] A. Jane, R. Dronov, A. Hodges, and N. H. Voelcker, "Porous silicon biosensors on the advance," Trends in Biotechnology, vol. 27, no. 4, pp. 230-239, 2009.

[30] D. Nilsson, T. Kugler, P.-O. Svensson, and M. Berggren, "An all-organic sensor-transistor based on a novel electrochemical transducer concept printed electrochemical sensors on paper," Sensors and Actuators, B: Chemical, vol. 86, no. 2-3, pp. 193-197, 2002.

[31] B. Meier, L. Egermann, S. Voigt, M. Stanel, H. Kempa, and A. C. Huebler, "Drift in the resistance of poly(3,4-ethylenedioxythiophene): poly(styrenesulfonate) printed films during thermal cycling," Thin Solid Films, vol. 519, no. 19, pp. 6610-6612, 2011.

[32] J. Salonen, M. Björkqvist, E. Laine, and L. Niinistö, "Stabilization of porous silicon surface by thermal decomposition of acetylene," Applied Surface Science, vol. 225, no. 1-4, pp. 389394, 2004.

[33] J. Tuura, M. Björkqvist, J. Salonen, and V.-P. Lehto, "Electrically isolated thermally carbonized porous silicon layer for humidity sensing purposes," Sensors and Actuators B: Chemical, vol. 131, no. 2, pp. 627-632, 2008.

[34] J. Sarfraz, A. Määttänen, P. Ihalainen, M. Keppeler, M. Lindén, and J. Peltonen, "Printed copper acetate based $\mathrm{H}_{2} \mathrm{~S}$ sensor on paper substrate," Sensors and Actuators B: Chemical, vol. 173, pp. 868-873, 2012.

[35] J. Sarfraz, P. Ihalainen, A. Määttänen et al., "Stable ink dispersions suitable for roll-to-roll printing with sensitivity towards hydrogen sulphide gas," Colloids and Surfaces A: Physicochemical and Engineering Aspects, vol. 460, pp. 401-407, 2014.

[36] A. Dwan, "Paper complexity and the interpretation of conservation research," Journal of the American Institute for Conservation, vol. 26, no. 1, pp. 1-17, 1987.

[37] A. K. Ghosh, "Fundamentals of paper drying-theory and application from industrial perspective," in Evaporation, Condensation and Heat transfer, A. Ahsan, Ed., chapter 25, InTech, 2011.

[38] R. Zhang, M. Gu, and G. Chen, "Non-isothermal crystallization kinetics of kaolin modified polyester," Journal Wuhan University of Technology, Materials Science Edition, vol. 26, no. 5, pp. 945949, 2011.

[39] Y.-F. Chen, M.-C. Wang, and M.-H. Hon, "Phase transformation and growth of mullite in kaolin ceramics," Journal of the European Ceramic Society, vol. 24, no. 8, pp. 2389-2397, 2004.

[40] V. Sanchez-Romaguera, M. A. Ziai, D. Oyeka et al., “Towards inkjet-printed low cost passive UHF RFID skin mounted tattoo paper tags based on silver nanoparticle inks," Journal of Materials Chemistry C, vol. 1, no. 39, pp. 6395-6402, 2013. 
[41] A. Määttänen, P. Ihalainen, P. Pulkkinen, S. Wang, H. Tenhu, and J. Peltonen, "Inkjet-printed gold electrodes on paper: characterization and functionalization," ACS Applied Materials and Interfaces, vol. 4, no. 2, pp. 955-964, 2012.

[42] K. Ankireddy, M. Iskander, S. Vunnam, D. E. Anagnostou, J. Kellar, and W. Cross, "Thermal analysis of silver nanoparticles for flexible printed antenna fabrication," Journal of Applied Physics, vol. 114, no. 12, Article ID 124303, 2013.

[43] L. L. Schramm, The Language of Colloid \& Interface Science, ACS Professional Reference Book, ACS, Washington, DC, USA, 1993. 

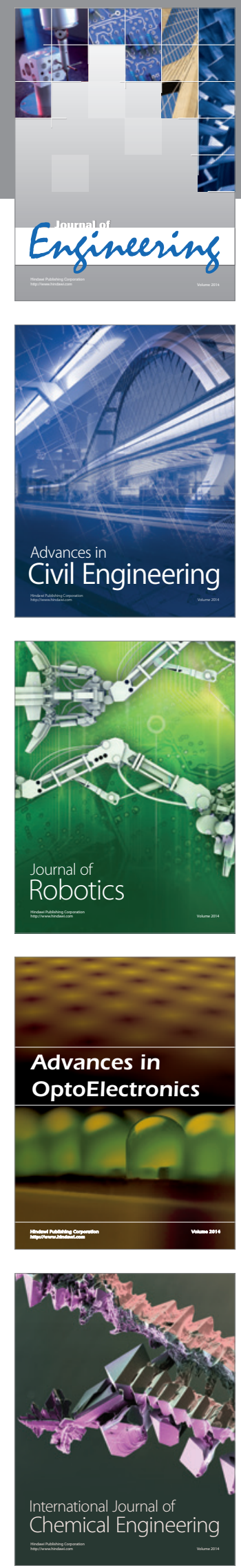

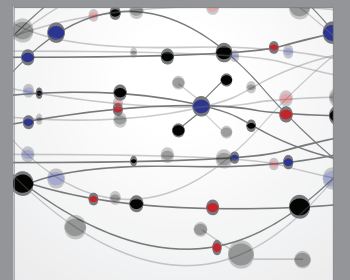

The Scientific World Journal
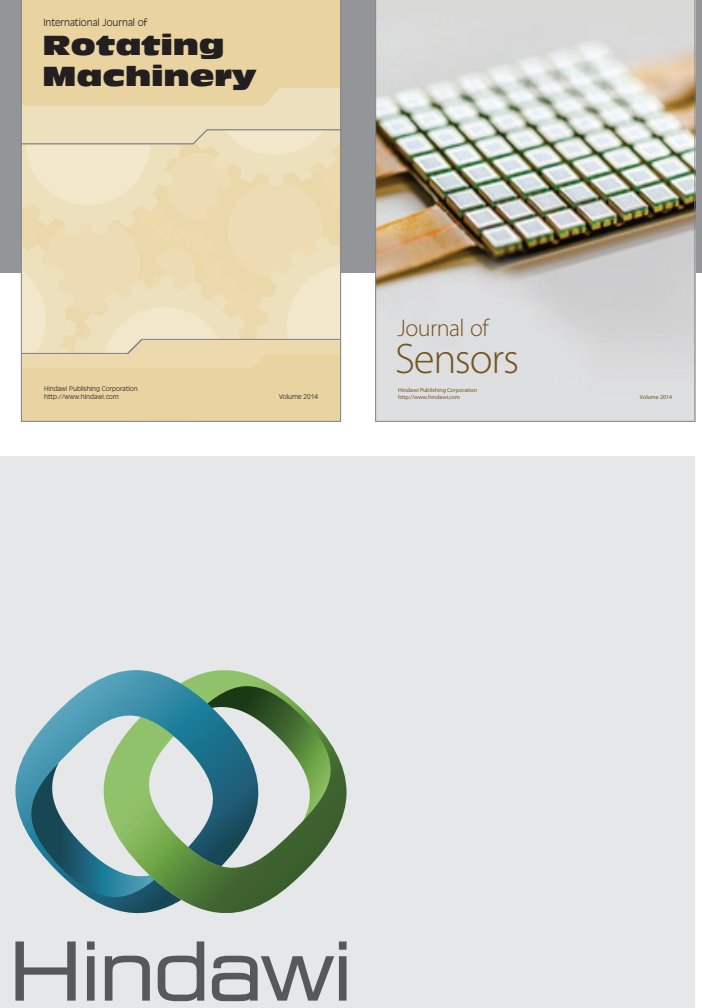

Submit your manuscripts at http://www.hindawi.com
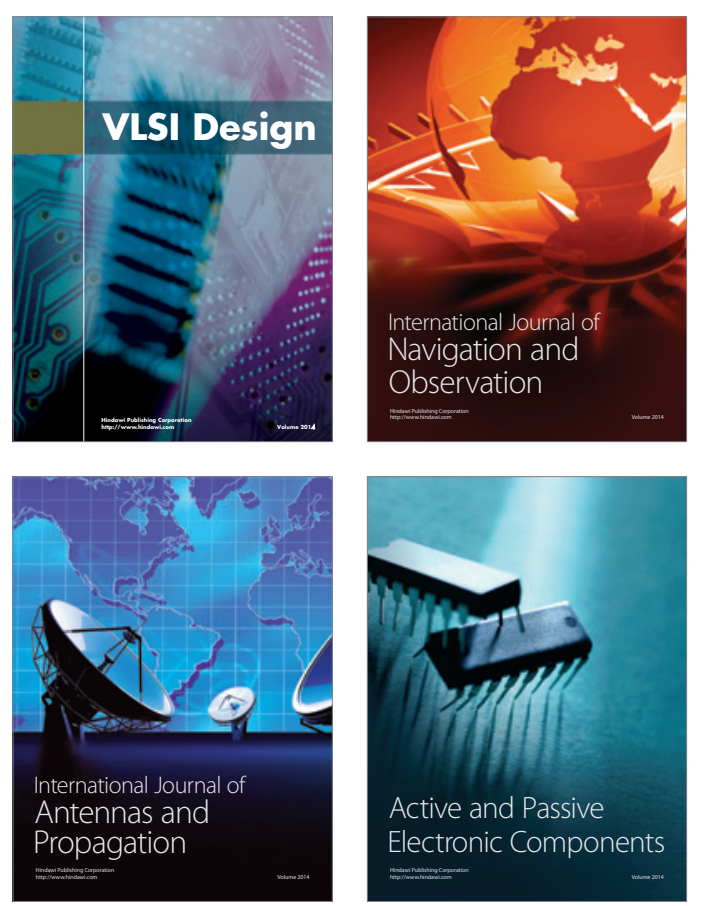
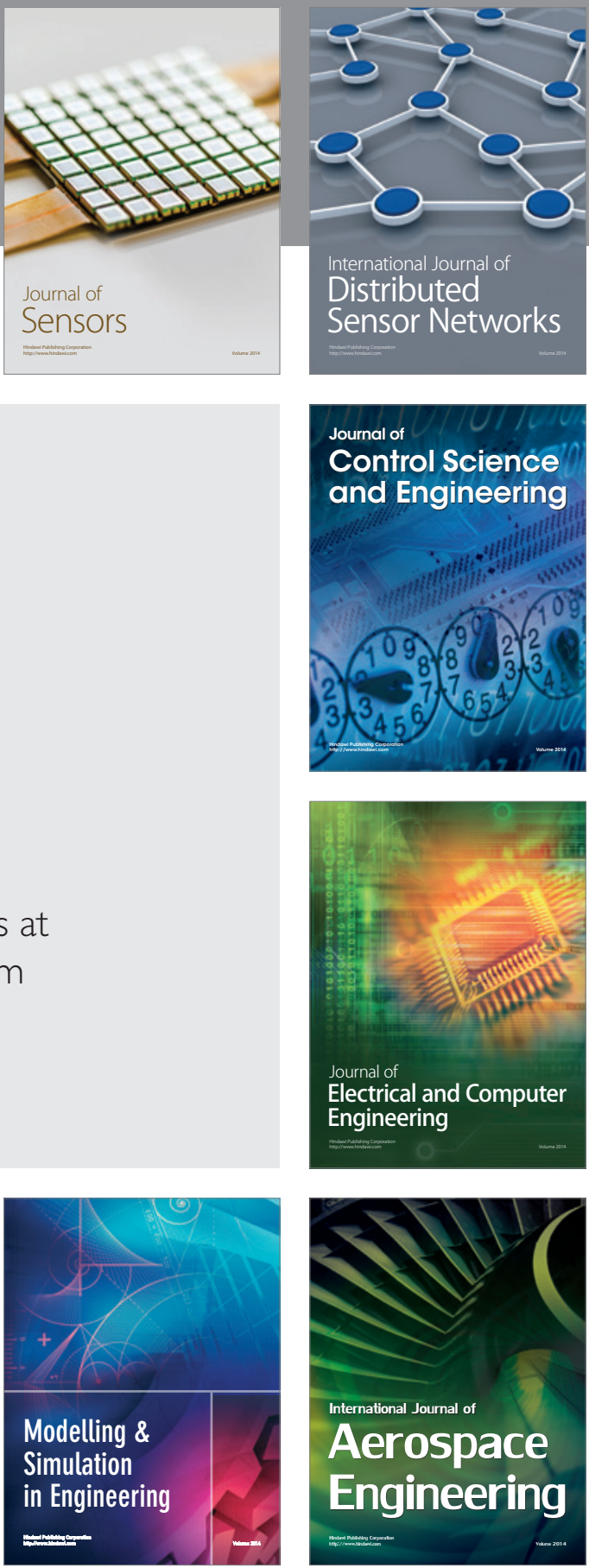

Journal of

Control Science

and Engineering
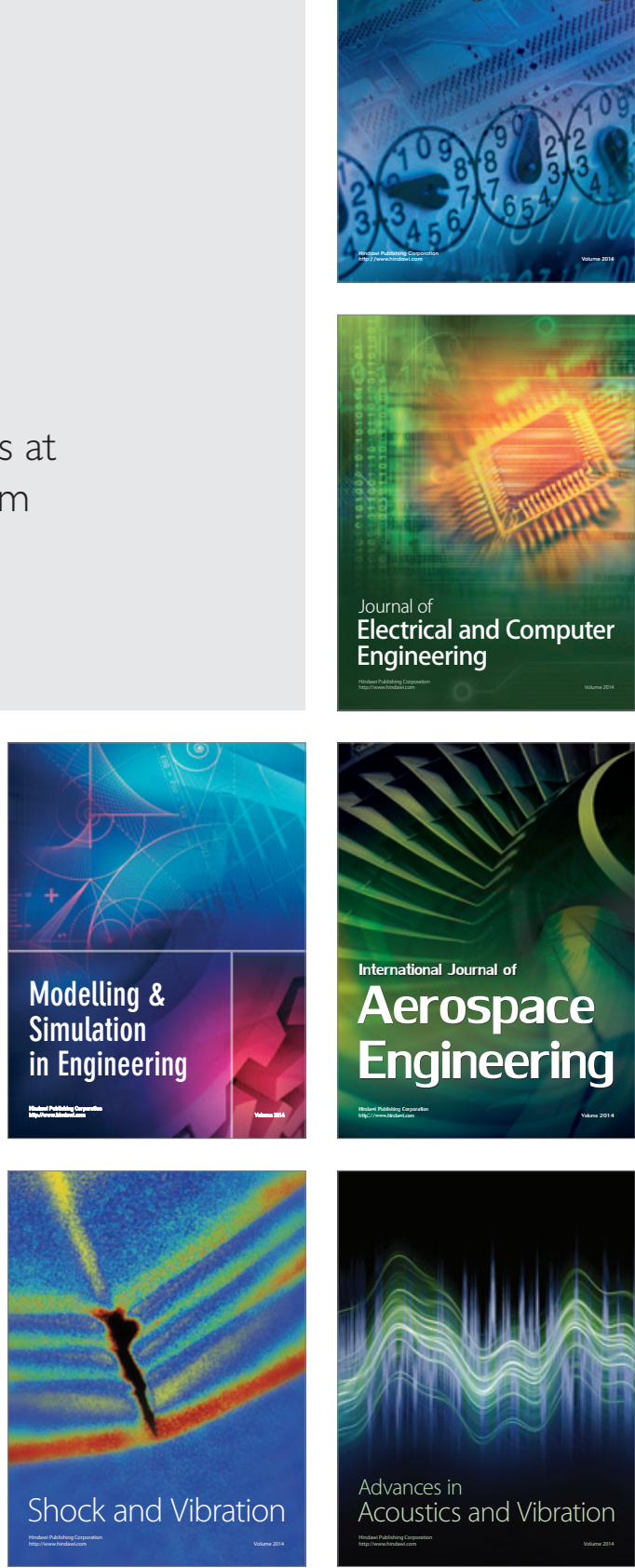